\title{
A Simple Algorithm to Eliminate Ambiguities in EBSD Orientation Map Visualization and Analyses: Application to Fatigue Cracks in Aluminum Alloys
}

\author{
Vipul K. Gupta* and Sean R. Agnew** \\ *National Institute of Aerospace, Hampton, VA 23666 \\ **Dept. of Materials Science and Engineering, University of Virginia, Charlottesville, VA 22904
}

An average value of automatically collected lattice orientations from several locations within a grain allows one to precisely define and analyze the orientation spread and spatial distribution of misorientation angle within a grain. A number of averaging methods, e.g. the mean or median orientation, or a quaternion-average [e.g., 1,2] from a set of near-orientations, have been proposed. Notably, such averaging methods suffer inaccuracies, if dataset contains symmetrically equivalent crystal orientations. Thus, when evaluating the average orientation of a given dataset, it is necessary to find an orientation cloud with pixel orientations that result in minimum misorientation between each pair. Additionally, for any choice of unique orientation sub-space, a set of similar orientations may cross a symmetry boundary (hereafter wrap-around effect) [3]. This means, an orientation dataset may have orientation subsets that are far apart in terms of their angular values despite small misorientation angle between any given pair. The all-Euler angle (AE) mapping scheme does not always provide an intuitive relationship between specific colors and their corresponding orientations when one or more of the Eulerian angles, is near a symmetry boundary, causing striking color differences where little or no significant orientation difference exists (see, e.g. AE map in Fig. 1a.) Owing to this situation, the inverse pole-figure (IFP) based coloring scheme is preferred, since it is less susceptible to the wrap-around color variations. However, it may suffer from ambiguities as well. For instance, grains with identical crystallographic axes $<111>,<110>$ and $<100>$ parallel to a specified IPF projection direction will have the same color on IPF map (Fig. 1b), but may have significantly different orientations. During our own work, on fatigue cracking in aluminum alloys [4], many cases of crystal-symmetry and wrap-around induced ambiguities were observed. This motivated us to develop and implement a simple algorithm to eliminate ambiguities, in both visualization and statistical analyses of EBSD orientation data.

Using crystal symmetry operators and the lowest Euclidian-distance criterion, the orientation of each pixel within a grain is redefined (see the result, e.g. AE map in Fig. 1c.). This turns all pixel orientations to the center of a prescribed Euler sub-space and, thus, eliminates the wrap-around effect on orientation dataset. An advantage of this approach is demonstrated, through a couple of examples, for direct determination of the representative orientation of a grain within an EBSD map by mean, median or quaternion-based averaging methods (e.g. Fig. 1e) that can be further used within analyses or visualization of misorientation spread or geometrically necessary dislocation (GND) density within a grain. The direct mapping of the intra-grain misorientation (Fig. 2a) with respect to the representative orientation clearly delineates the large plastic damage accumulation near the crack-tip. If one also considers the lattice curvature tensor, five components of the dislocation density tensor [5] - corresponding to a part of the GND content - may be inferred (e.g., see the distribution of estimated GND density in Fig. 2b.)

\section{References}

[1] K. Kunze et al., Texture and Microstructures, 20 (1993) 41-54.

[2] M. Humbert et al., J. Appl. Cryst. 29 (1996) 662-666. 
[3] J.C. Glez and J. Driver, J. Appl. Cryst. 34 (2001) 280-288.

[4] V.K. Gupta, Ph.D. Dissertation, University of Virginia, Charlottesville, 2009.

[5] W. Pantleon, Scripta Mater. 58 (2008) 994-997.

This research was supported by the Air Force Office of Scientific Research (F49620-03-1-0155) with Drs. Hartley, Tiley, and Conner as program managers, and by the Defense Advanced Research Projects Agency under contract HR0011-04-C-0003 with Dr. Christodoulou as the DARPA program manager and Dr. John Papazian (dec) as the Northrop Grumman technical manager.

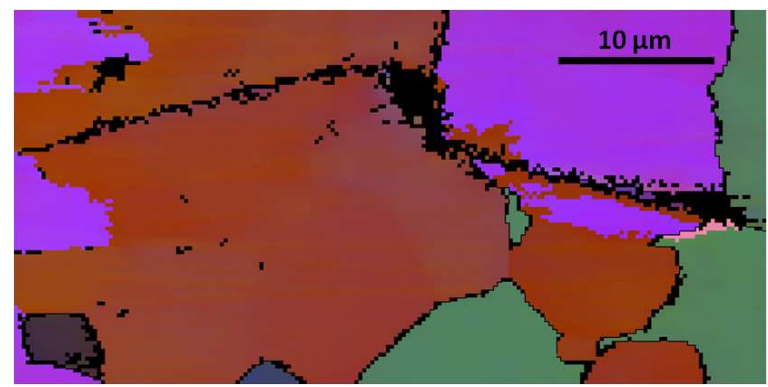

(a)

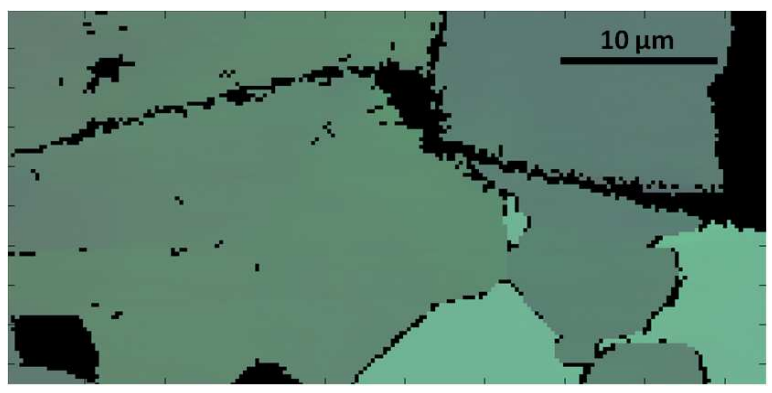

(c)

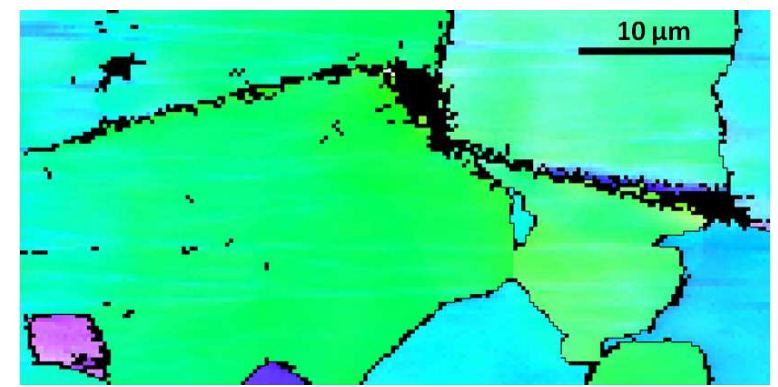

(b)

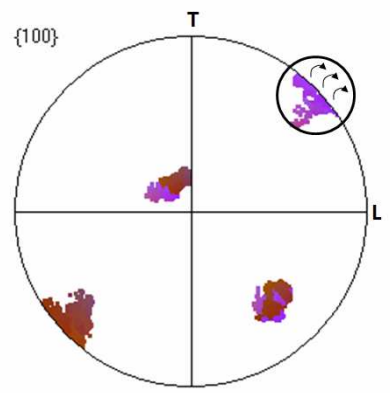

(d)

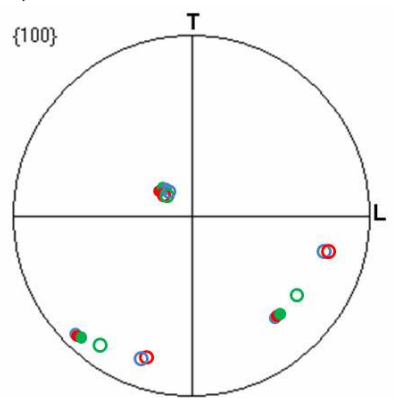

(e)

FIG. 1. a) \& b) EBSD AE and IPF maps (as-collected), c) AE map after applying the present algorithm, d) \& e) $\{100\}$ pole-figures. Fig. 1e illustrates the positions of mean, median and quaternion-average orientations determined from the as-collected orientation dataset (RGB, open symbols) and after applying the present algorithm (RGB, solid symbols), respectively. Note the distribution of average orientations in the former case due to the wrap-around effect.

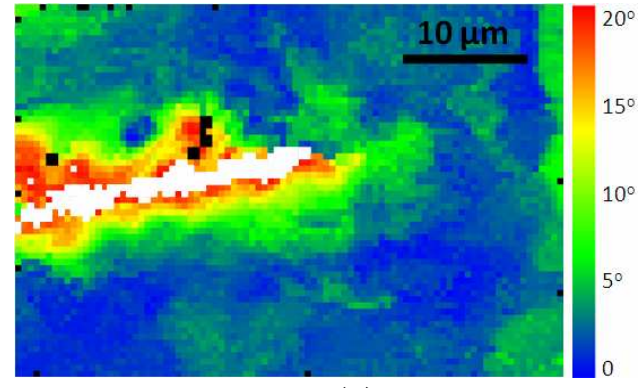

(a)

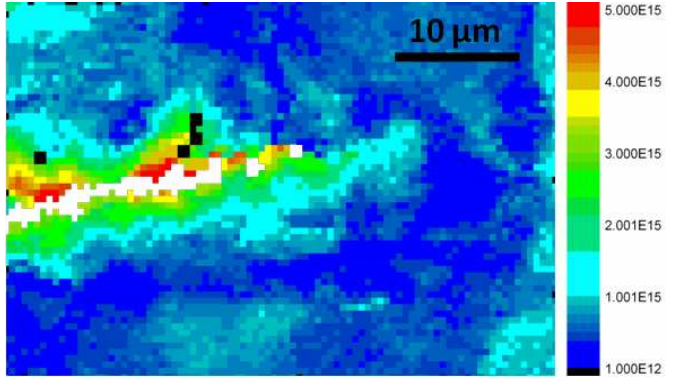

(b)

FIG. 2. Misorientation spread relative to the quaternion-average (determined from the redefined orientation dataset), at a crack-tip, b) GND density map estimated from the EBSD orientation data. Color-bars on the right side of Figs. $2 \mathrm{a} \& \mathrm{~b}$ illustrate the relative amount of misorientation from the average orientation and the dislocation density contained within each measured pixel, respectively. 